\title{
Scaled Bilateral Teleoperation Using Discrete-Time Sliding-Mode Controller
}

\author{
Shahzad Khan, Member, IEEE, Asif Sabanovic, Senior Member, IEEE, and Ahmet Ozcan Nergiz
}

\begin{abstract}
In this paper, the design of a discrete-time slidingmode controller based on Lyapunov theory is presented along with a robust disturbance observer and is applied to a piezostage for high-precision motion. A linear model of a piezostage was used with nominal parameters to compensate the disturbance acting on the system in order to achieve nanometer accuracy. The effectiveness of the controller and disturbance observer is validated in terms of closed-loop position performance for nanometer references. The control structure has been applied to a scaled bilateral structure for the custom-built telemicromanipulation setup. A piezoresistive atomic force microscope cantilever with a built-in Wheatstone bridge is utilized to achieve the nanonewtonlevel interaction forces between the piezoresistive probe tip and the environment. Experimental results are provided for the nanonewton-range force sensing, and good agreement between the experimental data and the theoretical estimates has been demonstrated. Force/position tracking and transparency between the master and the slave has been clearly demonstrated after necessary scaling.
\end{abstract}

Index Terms-Bilateral control, discrete sliding-mode control (SMC), disturbance observer, force sensing, high-precision motion control, telemicromanipulation.

\section{INTRODUCTION}

I N RECENT years, the demand for microsystem technology has grown rapidly, particularly due to the development of microelectromechanical systems (MEMS) products such as accelerometers, inkjet printer heads, optical MEMS, etc. Complex micro/nano systems generally contain much distinct functionality in a single product and consist of different types of materials. Use of a monolithic (uniform) process to produce complex micro/nano systems is desirable but, unfortunately, is not always feasible. The current state of the art is to incorporate multiple incompatible components into a single product by utilizing serial assembly techniques, i.e., handling the parts one by one [1], [2]. The first and foremost requirement for the assembly process is to "precisely manipulate" objects. Manipulation includes cutting, pushing, pulling, indenting, or any other type of interactions that changes the relative position and relation of

Manuscript received November 4, 2007; revised April 9, 2008, September 10, 2008, and December 26, 2008. First published April 7, 2009; current version published August 12, 2009. This work was supported in part by the Yousef Jameel Scholarship and in part by the Scientific and Technological Research Council of Turkey (TUBITAK), Ankara, Turkey.

S. Khan is with the Department of Precision and Microsystems Engineering, Delft University of Technology, 2828 CD Delft, The Netherlands (e-mail: s.khan@tudelft.nl; shahzadfkt@yahoo.co.uk).

A. Sabanovic is with Sabanci University, Istanbul 34956, Turkey.

A. O. Nergiz is with the NSF Center for High-Rate Nanomanufacturing, Northeastern University, Boston, MA 02115 USA.

Color versions of one or more of the figures in this paper are available online at http://ieeexplore.ieee.org.

Digital Object Identifier 10.1109/TIE.2009.2018538 entities. The process of manipulation is very cumbersome to be performed autonomously with robotic systems and/or directly by human operators. Bilateral control, which is typically used for teleoperation, offers a solution to these tasks since it enables the operator to work remotely without actually being there. That is, if the actual presence of an operator is not possible, inclusion of a bilateral control system between the operator and the task would simply give a possibility to the so-called "telepresence" of the operator.

Bilateral control is defined as the control of two systems working together on an actual or virtual task. Typically, it is used for teleoperation, in which one system is called the "master" side and the other is called the "slave" side of bilateral action. The slave subsystem tracks the positions of the master subsystem, and the master side provides the forces encountered by the slave side to the operator, and hence, teleoperation is achieved [3], [4]. Nowadays, many researchers have come up with the notion of "multilateral control" [5], [6] consisting of more than two systems working with proper coordination to achieve a desired task. In order to perform telemicromanipulation, it is indispensable to achieve robust and transparent bilateral controllers for human intervention so that high-fidelity position/force interaction between the operator and the remote micro/nano environment can be achieved [7], [8]. As bilateral control enables skilled teleoperation on several tasks, it offers better safety, low cost, and high accuracy. On the other hand, it also suffers from time delay problem [9], [10] that effects the transparency of the systems.

For high-precision motion control problems, robustness of the control algorithm is the most crucial element, even if the system model is linear. Furthermore, when the plant to be controlled has nonlinearities such as internal hysteresis, which is the case for lead zirconate titanate (PZT, also known as piezoactuator), then the advantage of designing a robust controller according to nominal plant parameters to reject parameter uncertainties would provide less effort than modeling the system and utilizing disturbance compensation methods [11], [12]. Moreover, it is a fact that using more complicated models may not always lead to better disturbance compensation results than just using a simple model (e.g., the model of Coulomb friction) since the quality of disturbance compensation depends not only on the model but also on the implementation constraints.

To avoid the difficulties mentioned earlier and concentrate on the main issues of the control problem, one needs to find a methodology that produces a robust controller design according to the nominal parameters and has fine disturbance rejection to achieve high-precision motion control with minimum effort. As performance requirements become more stringent, classical 
controllers such as proportional-integral-derivative, which has been the most preferred controller and widely used in industry for generations, can no longer provide acceptable results. The theory of variable-structure systems (VSSs) has opened up a wide new area of development for control designers. Variablestructure control (VSC), which is frequently known as slidingmode control (SMC), is characterized by a discontinuous control action that changes structure upon reaching a set of predetermined switching surfaces. Some of the concepts and theoretical advances of VSS are covered by Young et al. [13], Utkin [14], and Sabanovic et al. [15]. These kinds of control may result in a very robust system and provide a possibility for achieving the goals of high-precision motion. The SMC theory was initially developed from a continuous-time perspective. It has been realized that directly applying continuous-time SMC algorithms to discrete-time systems will lead to some indomitable problems, such as limited sampling frequency, sample/hold effects, and discretization errors. Since the switching frequency in sampled-data systems cannot exceed the sampling frequency, a discontinuous control will disable generation of motion in a random manifold in discrete-time systems. This leads to chattering at the sampling frequency along the designed sliding surface or even instability in case of a too large switching gain. In order to cope with the aforementioned issues, a discrete-time SMC structure has been derived.

In order to achieve force transparency between the master and the slave, it is necessary to sense the force in the nanonewton range with high accuracy. Many researchers have used different ways for sensing or estimating force using PZT actuators [16], capacitive sensors, optical deflection as in the atomic force microscope (AFM) scheme, tunneling as in scanning tunneling microscope, etc. A piezoresistive AFM cantilever with a builtin Wheatstone bridge is utilized as a force sensor. Piezoresistive sensors have been used for many other MEMS applications, including accelerometers, gyroscopes, and AFM cantilevers.

In this paper, the design and implementation of a discrete sliding-mode controller, along with a disturbance observer, based on SMC are presented to eliminate nonlinear disturbances acting on PZT in order to achieve high position accuracy in the nanoscale. Moreover, force sensing with the nanonewton range using a piezoresistive AFM cantilever is presented, and finally, the force/position tracking of the master and the slave for the telemicromanipulation setup is achieved.

This paper is organized as follows. Section II explains the telemicromanipulation setup, Section III discuss the design of the discrete sliding-mode controller, and Section IV focuses on modeling of PZT actuator along with the simulation and experimental results for position control using SMC. Section V discusses scaled bilateral teleoperation using SMC, including the experimental validation of force sensing with nanonewton accuracy, and Section VI shows the experimental results of the force/position tracking of the master and the slave. Section VII describes the conclusion.

\section{Telemicromanipulation Setup}

The system is composed of two parts, namely, a master mechanism operated by the human operator and a slave mech-

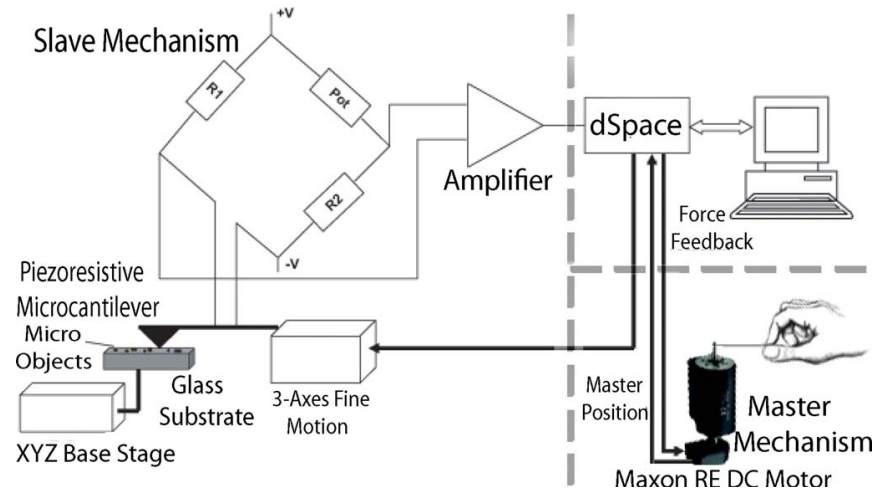

Fig. 1. Schematic view of the telemicromanipulation system.

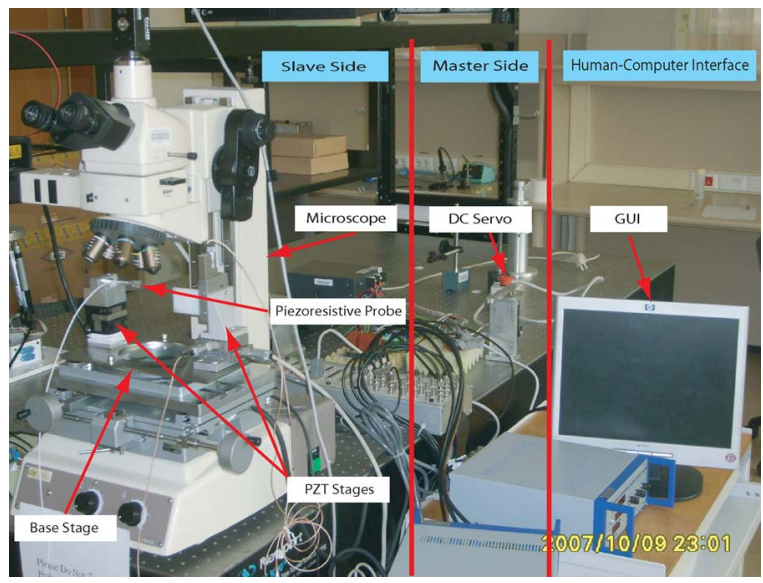

Fig. 2. Experimental setup for telemicromanipulation.

anism interacting with the micro environment. For the master mechanism, a dc servo is utilized, while a piezoresistive microcantilever attached on PZT stacks is used for the slave. A bilateral man-machine interface is implemented for control, as shown by schematics in Fig. 1.

The position data from the master side are scaled and transferred to the slave side, while simultaneously, the force measured at the slave side is scaled and transferred back to the master. XYZ base stages are manually operated for proper alignment of the microobject under the workspace. A graphical display is also made available to the operator. Fig. 2 shows the experimental setup.

An open architecture telemicromanipulation system that satisfies the requirements has been developed and used as the slave mechanism. Nanoscale positioning of the microcantilever has been provided using three-axis piezostages (P-611 by Physik Instrumente), which are driven by a power amplifier (E-664) in closed-loop external control mode. The strain gauge sensors integrated in the amplifier are utilized for position measurement of the closed-loop stages that possess a travel range of $100 \mu \mathrm{m} / \mathrm{axis}$ with 1-nm theoretical resolution. An open-loop piezoelectric micrometer drive (PiezoMike PI-854 from Physik Instrumente) has been utilized as the base stage, which is equipped with integrated high-resolution piezo linear drives [17]. Manually operable linear drives are capable of $1-\mu \mathrm{m}$ resolution, and the automatic movement range of the 
micrometer tip with respect to the position can be set to $50 \mu \mathrm{m}$ (25 $\mu \mathrm{m} \mathrm{in/out).} \mathrm{As} \mathrm{for} \mathrm{the} \mathrm{force} \mathrm{feedback,} \mathrm{a} \mathrm{piezoresistive}$ AFM cantilever with a built-in Wheatstone bridge from Applied NanoStructures, Inc., has been utilized. A real-time controller card (dSPACE DS1103) is used as a control platform along with an optical microscope (Nikon MM-40) which is used as visual feedback for the human operator.

\section{Sliding Mode in VARIable-Structure Systems}

SMC, which is sometimes known as VSC, is characterized by a discontinuous control action that changes structure upon reaching a set of predetermined switching surfaces. This control structure may result in a very robust system and thus provides a possibility for achieving the goals of high-precision motion. Consider the following system:

$$
\dot{x}=f(x, t)+B(x, t) u(x, t), \quad x \subset R^{n}, u \subset R^{m}
$$

where all the elements and their first-order time derivatives of vector $f(x, t)$ and matrix $B(x, t)$ are continuous and bounded. The rank of $B(x, t)=m \forall x, t>0$.

The discontinuous control is given by

$$
\begin{aligned}
u & = \begin{cases}u^{+}(x, t), & \sigma(x)>0 \\
u^{-}(x, t), & \sigma(x)<0\end{cases} \\
\sigma(x)^{T} & =\left\{\sigma_{1}(x), \sigma_{2}(x), \ldots, \sigma_{m}(x)\right\} \\
\sigma(x) & =G\left(x^{r}-x\right)
\end{aligned}
$$

where $u^{+}(x, t), u^{-}(x, t)$, and $\sigma(x)$ are continuous functions; $G$ is a positive integer chosen for the error-converging response time; and $x^{r}$ is the reference position. $u(x, t)$ undergoes discontinuity on the manifold $\sigma=0$.

Let $S=\left.X\right|_{\sigma(x)=0}$ be a switching manifold that includes the origin $x=0$. If, for any $x_{0}$ in $S, x(t)$ is in $S$ for all $t>t_{0}$, then $x(t)$ is a sliding mode of the system, and the manifold $S$ is called a sliding manifold. A sliding mode exists if, in the vicinity of the switching surface $S$, the tangent or the velocity vectors of the state trajectory always point toward the switching surface.

\section{A. Design of Discrete Sliding-Mode Controller}

Drakunov and Utkin [18] introduced a continuous approach to SMC for an arbitrary finite dimensional discrete-time system. This approach implies that, for a sampled-data controller (as the system becomes discrete), the controller should be continuous to overcome the sampling frequency limitations of the discontinuous approach. For a such continuous implementation of SMC, plant motion is proven to reach the sliding manifold of the predefined state trajectory in finite time.

The derivation of the controller structure can be achieved using proper selection of the Lyapunov function $V(\sigma)$ and an appropriate form of the derivatives of the Lyapunov function $\dot{V}(\sigma)$. The system under consideration in this paper is single input-single output, so further derivation will be carried out for systems with scalar input and scalar sliding-mode function $\sigma(x) \subset \Re^{1}$.

A suitable candidate of the Lyapunov function can be taken as

$$
V(\sigma)=\frac{\sigma^{2}}{2} .
$$

Hence, the derivative of the Lyapunov function is

$$
\dot{V}(\sigma)=\sigma \dot{\sigma} .
$$

In order to guarantee the asymptotic stability of the solution $\sigma\left(x, x^{r}\right)=0$, the derivatives of the Lyapunov function may be selected to be

$$
\dot{V}(\sigma)=-D \sigma^{2}-\mu \frac{\sigma^{2}}{|\sigma|} .
$$

Here, $D$ and $\mu$ are positive constants. Hence, if the control can be determined from (5) and (6), then the asymptotic stability will be guaranteed since $V(\sigma)>0, V(0)=0$, and $\dot{V}(\sigma)<0$. By combining (5) and (6), the following equation can be deduced, i.e., $\sigma(\dot{\sigma}+D \sigma+\mu(\sigma /|\sigma|))=0$, which results in (7)

$$
\dot{\sigma}+D \sigma+\mu \frac{\sigma}{|\sigma|}=0 \text {. }
$$

The derivative of the sliding function can be written as $\dot{\sigma}=G\left(\dot{x}^{r}-\dot{x}\right)=G \dot{x}^{r}-G \dot{x}$. Here, $G=\{\lambda 1\}$, with $\lambda$ being a positive constant. After some simplification, $\dot{\sigma}$ can be written as $\dot{\sigma}=G \dot{x}^{r}-G f-G B u(t)=G B\left(u_{\mathrm{eq}}-u(t)\right)$, and can be further deduced to (8)

$$
u(t)=u_{\mathrm{eq}}+(G B)^{-1}\left(D \sigma+\mu \frac{\sigma}{|\sigma|}\right) .
$$

Direct implementation of control (8) requires knowledge of the equivalent control $u_{\mathrm{eq}}=(G B)^{-1} G f$, which is unlikely. The discrete-time implementation of control is most likely, so we will show an approach that allows approximation of term $u_{\mathrm{eq}}$ and leads to simple recursive implementation of the control algorithm (8).

Assuming sampling interval $T_{s}$ and $k=Z^{+}$, by applying Euler approximation from $\dot{\sigma}=(G B)\left(u_{\mathrm{eq}}-u\right)$, one can write

$$
\sigma\left((k+1) T_{s}\right)=\sigma\left(k T_{s}\right)+T_{s}(G B)\left(u_{\mathrm{eq}}\left(k T_{s}\right)-u\left(k T_{s}\right)\right) .
$$

The discretized control (8) may be written as

$$
u\left(k T_{s}\right)=u_{\mathrm{eq}}\left(k T_{s}\right)+(G B)^{-1}\left(D \sigma\left(k T_{s}\right)+\mu \frac{\sigma\left(k T_{s}\right)}{\left|\sigma\left(k T_{s}\right)\right|}\right) .
$$

The $u_{\text {eq }}\left(k T_{s}\right)$ needed for implementation of the discretized control (10) could be determined from (9), but that would require estimation of $\sigma\left((k+1) T_{s}\right)$. In order to avoid usage of the predicted value $\sigma\left((k+1) T_{s}\right)$ in the calculation of control input, an approximation of the equivalent control $u_{\mathrm{eq}}\left(k T_{s}\right)$ could be used. The equivalent control $u_{\mathrm{eq}}=(G B)^{-1} G f$ is continuous, so it may be approximated with the order of the one sampling 
period $O\left(T_{s}\right)$ by taking a single-step backward approximation and can be written as $u_{\text {eq }}\left(k T_{s}\right) \cong u_{\text {eq }}\left((k-1) T_{s}\right)$. From (9), one can write $\sigma\left(k T_{s}\right)$ as

$$
\begin{aligned}
\sigma\left(k T_{s}\right) & =\sigma\left((k-1) T_{s}\right) \\
& +T_{s}(G B)\left(u_{\text {eq }}\left((k-1) T_{s}\right)-u\left((k-1) T_{s}\right)\right) .
\end{aligned}
$$

Consequently, by rearranging $(9), u_{\mathrm{eq}}\left((k-1) T_{s}\right)$ may be expressed in the following form:

$$
\begin{aligned}
u_{\mathrm{eq}}\left((k-1) T_{s}\right) & =u\left((k-1) T_{s}\right) \\
+ & (G B)^{-1} \frac{1}{T_{s}}\left(\sigma\left(k T_{s}\right)-\sigma\left((k-1) T_{s}\right)\right) .
\end{aligned}
$$

By inserting (11) into (10), the approximated control [with approximation error being $\left.u_{\mathrm{eq}}\left(k T_{s}\right)-u_{\mathrm{eq}}\left((k-1) T_{s}\right)=O\left(T_{s}\right)\right]$ may be expressed as

$$
\begin{aligned}
u\left(k T_{s}\right) \cong & u_{\mathrm{eq}}\left((k-1) T_{s}\right)+(G B)^{-1}\left(D \sigma\left(k T_{s}\right)+\mu \frac{\sigma\left(k T_{s}\right)}{\left|\sigma\left(k T_{s}\right)\right|}\right) \\
= & u\left((k-1) T_{s}\right)+(G B)^{-1} \frac{1}{T_{s}} \\
& \times\left(\left(1+D T_{s}\right) \sigma\left(k T_{s}\right)-\sigma\left((k-1) T_{s}\right)+T_{s} \mu \frac{\sigma\left(k T_{s}\right)}{\left|\sigma\left(k T_{s}\right)\right|}\right) .
\end{aligned}
$$

The control structure (13) is suitable for implementation since it requires measurement of the sliding-mode function and the value of the control applied in the preceding step. For a discrete-time system, the discrete sliding mode can be interpreted as the only state that is required to be kept on the sliding surface at each sampling instant. Between the samples, the states are allowed to diverge from the surface within a boundary layer, and estimation of the boundary layer will be explained in next section.

\section{B. Estimation of Boundary Layer in Discrete SMC}

During the course of designing a controller, it is crucial to analyze the robustness of the controller or, in other words, whether it satisfies the condition defined by (7). Moreover, estimation of the boundary layer of the sliding manifold is significant in relation with the robustness of the controller. The analysis shown is concerned with a general system as in (1), where $f(x, t)$ and $B(x, t)$ are assumed to be continuous and bounded. The derivative of the sliding surface is given by

$$
\begin{aligned}
\frac{d \sigma(t)}{d t} & =G\left(\dot{x}^{r}-\dot{x}\right)=G \dot{x}^{r}(t)-G f(t)-G B u(x, t) \\
& =G B\left(u_{\mathrm{eq}}(t)-u(t)\right)
\end{aligned}
$$

The intersampling change of $\sigma(t)$ may be evaluated by inserting a control from (13) into (14). Assume that the following result is obtained:

$$
\begin{aligned}
\frac{d \sigma(t)}{d t}= & G B\left(u_{\mathrm{eq}}(t)-u\left(t^{-}\right)\right) \\
= & G B\left(u_{\mathrm{eq}}(t)-u_{\mathrm{eq}}\left((k-1) T_{s}\right)\right. \\
& \left.\quad-(G B)^{-1}\left(D \sigma\left(k T_{s}\right)+\mu \frac{\sigma\left(k T_{s}\right)}{\left|\sigma\left(k T_{s}\right)\right|}\right)\right) \\
= & G B\left(u_{\mathrm{eq}}(t)-u_{\mathrm{eq}}\left((k-1) T_{s}\right)\right) \\
& -\left(D \sigma\left(k T_{s}\right)+\mu \frac{\sigma\left(k T_{s}\right)}{\left|\sigma\left(k T_{s}\right)\right|}\right) .
\end{aligned}
$$

After simplification, (15) can be written as

$$
\begin{aligned}
\frac{d \sigma(t)}{d t}+D \sigma(t) & =G B \Delta u_{\mathrm{eq}}(t)-D \Delta \sigma-D \mu=\xi(t) \\
& =G B \Delta u_{\mathrm{eq}}(t)-G B \Delta x^{r}-D G \Delta x-D \mu \\
& =\xi(t) .
\end{aligned}
$$

The terms can be written as

$$
\begin{aligned}
\Delta u_{\mathrm{eq}}(t) & =u_{\mathrm{eq}}\left((k-1) T_{s}\right)=O\left(T_{s}\right) \\
\Delta \sigma & =\sigma(t)-\sigma\left(k T_{s}\right) .
\end{aligned}
$$

Since $u_{\mathrm{eq}}(t), x(t)$, and $x^{r}(t)$ are continuous functions, then its order depends on the selection of $\mu$. By selecting $\mu$ small enough $\xi(t)$ becomes in the order of the sampling period, i.e., $\xi(t)=O\left(T_{s}\right)$ and the boundary layer is then of the order $O\left(T_{s}^{2}\right)$.

\section{High-Precision Motion Using Piezoactuator}

\section{A. Modeling PZT Actuator}

Since piezoceramic is a known dielectric, one would expect a PZT stack actuator to exhibit a capacitive behavior along with the rate-independent hysteresis exhibited, which affects the net electrical charge delivered to the actuator. Additionally, dynamic observation indicates that endpoint displacement as a function of electrical charge is well approximated by secondorder linear dynamics.

The piezostage consists of a piezodrive with a flexure-guided structure, which is designed to possess zero stiction and friction. Moreover, the flexure stages exhibit high stiffness, high load capacity, and shock and vibration insensitivity. Fig. 3 describes the overall electromechanical model [19] of a PZT actuator.

The hysteresis and piezoelectric effects are separated. $H$ represents the hysteresis effect, and $u_{h}$ is the voltage due to this effect. The piezoelectric effect is represented by $T_{\mathrm{em}}$, which is an electromechanical transducer with transformer ratio. The capacitance $C_{e}$ represents the sum of the capacitances of the individual PZT wafers, which are electrically in parallel. The total current flowing through the circuit is $\dot{q}$. Furthermore, $q$ may be seen as the total charge in the PZT actuator. The charge $q_{p}$ is the transducer charge from the mechanical side. The voltage $u_{p}$ is due to the piezoeffect. The total voltage over 

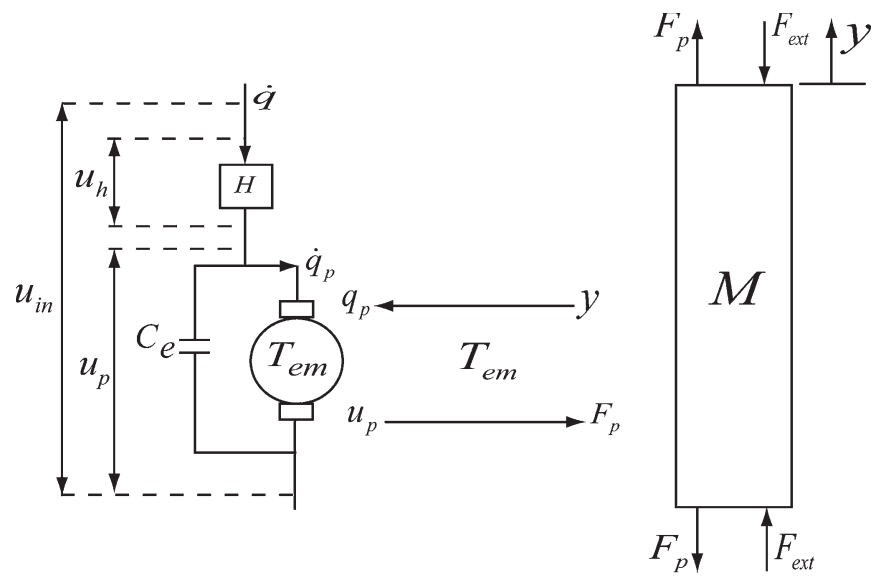

Fig. 3. Electromechanical model of a PZT actuator.

TABLE I

Properties of PIEZostage

\begin{tabular}{|c|c|c|}
\hline Symbol & Quantity & Value in SI \\
\hline$m_{p}$ & Nominal Mass & $1.5 \times 10^{-3} \mathrm{~kg}$ \\
\hline$c_{p}$ & Nominal Damping & $220 \frac{\mathrm{Ns}}{\mathrm{m}}$ \\
\hline$k_{p}$ & Nominal Stiffness & $300000 \frac{\mathrm{N}}{\mathrm{m}}$ \\
\hline$f_{r}$ & Resonant Frequency & $350 \mathrm{~Hz}$ \\
\hline$T_{e m}$ & Transformation Ratio & $0.3 \frac{\mathrm{N}}{\mathrm{V}}$ \\
\hline
\end{tabular}

the PZT actuator is $u_{\text {in }}, F_{p}$ is the transducer force from the electrical side, $F_{\text {ext }}$ is the externally applied force, and $y$ is the resulting elongation of the PZT actuator. The mechanical relation between $F_{p}$ and $y$ is denoted by $M$. Note that we have equal electrical and mechanical energies at the ports of interaction, i.e., $u_{p} q_{p}=F_{p} y$.

The piezoelectric ceramic has elasticity modulus $E$, viscosity $\eta$, and mass density $\rho$. Furthermore, the geometric properties of the PZT actuator are length $L$ and cross-sectional area $A_{p}$. Nominal mass $m_{p}$, nominal stiffness $k_{p}$, and damping coefficient $c_{p}$ can be calculated as $m_{p}=\rho A_{p} L, k_{p}=E A_{p} / L$, and $c_{p}=\eta A_{p} / L$.

The complete electromechanical equations can be written as

$m_{p} \ddot{y}+c_{p} \dot{y}+k_{p} y=T_{\mathrm{em}}\left(u_{\mathrm{in}}(t)-H\left(y, u_{\mathrm{in}}\right)\right)-F_{\mathrm{ext}}$.

Here, $y$ represents the displacement of the piezostage, and $H\left(y, u_{\text {in }}\right)$ denotes the nonlinear hysteresis, which is a function of $y$ and $u_{\text {in }}$. The properties of the utilized piezoelectric actuator are shown in Table I. Since modeling hysteresis and using compensation techniques are cumbersome processes due to their dependencies on many factors, thus the disturbance observer which is discussed in the following section will be used for compensation of hysteretic behavior in the system.

\section{B. Disturbance Observer Based on SMC}

There are several hindrances for high-precision motion that is highly nonlinear in nature and arises from several factors such as hysteresis, dead zone, saturation, backlash, etc., of the actuators and/or sensing devices, high parameter variations, and time delay. It might be possible to combine all the effects of these different kinds of disturbances on the plant response (i.e., observe their position) and provide a compensation for them as an addition to the controller output and use this sum as the plant input. This kind of compensation is called "disturbance compensation," and the observer used is called "disturbance observer."

The observer structure is deduced based on (18) under the assumption that all plant parameter uncertainties, nonlinearities, and external disturbances can be represented as a lumped disturbance. It is assumed that $y$ is the displacement and measurable and that $u_{t}$ is the input and also a measurable quantity

$$
\begin{aligned}
m_{p} \ddot{y}+c_{p} \dot{y}+k_{p} y & =T_{\mathrm{em}} u(t)-F_{\mathrm{dis}} \\
F_{\mathrm{dis}} & =\Delta T\left(u_{\mathrm{in}}+v_{h}\right)+\Delta m \ddot{y}+\Delta c \dot{y}+\Delta k y .
\end{aligned}
$$

Here, $m_{p}, c_{p}$, and $k_{p}$ are the nominal plant parameters, while $\Delta m, \Delta c, \Delta k$, and $\Delta T$ are the uncertainties associated with the plant parameters. Since $y$ and $u(t)$ are measurable quantities, the observer structure can be written in the following form:

$$
m_{p} \ddot{\hat{y}}+c_{p} \dot{\hat{y}}+k_{p} \hat{y}=T_{\mathrm{em}} u-T_{\mathrm{em}} u_{c}
$$

Here, $\hat{y}, \dot{\hat{y}}$, and $\ddot{\hat{y}}$ are the estimated position, velocity, and acceleration, respectively. $u$ is the plant control input, and $u_{c}$ is the observer control input, as shown in Fig. 4.

The estimated position $\hat{y}$ should be forced to track $y$. The derivation process of the SMC structure is also used for deriving the observer controller whose sliding manifold is defined as $\sigma_{\text {obs }}=\lambda_{\text {obs }}(y-\hat{y})+(\dot{y}-\dot{\hat{y}})$.

Here, $\lambda_{\text {obs }}$ is a positive constant. If $\sigma_{\text {obs }}$ is forced to become zero, then $\hat{y}$ should be forced to $y$. As described in the previous section, with the same analogy, it can be written as $\left(\dot{\sigma}_{\text {obs }}+\right.$ $\left.D_{\text {obs }} \sigma_{\text {obs }}\right)=0$, which guarantees $\sigma_{\text {obs }} \rightarrow 0$.

After some modification, the resulting equation can be written as

$$
(\ddot{y}-\ddot{\hat{y}})+\left(\lambda_{\mathrm{obs}}+D_{\mathrm{obs}}\right)(\dot{y}-\dot{\hat{y}})+\lambda_{\mathrm{obs}} D_{\mathrm{obs}}(y-\hat{y})=0 \text {. }
$$

It can be seen that the transient of the closed-loop system is defined by the roots $-\lambda_{\text {obs }}$ and $-D_{\text {obs }}$. From structure (20), it can be seen that the input matrix $B_{\text {obs }}$ and $G$ can be written as

$$
\begin{aligned}
B_{\mathrm{obs}} & =\left[\begin{array}{ll}
0 & -\frac{T_{\mathrm{em}}}{m_{p}}
\end{array}\right]^{\mathrm{T}} \\
G & =\left[\begin{array}{ll}
\lambda_{\mathrm{obs}} & 1
\end{array}\right] .
\end{aligned}
$$

Thus, after some simplification, the observer structure can be written as

$$
u_{c_{k}}=u_{c_{k-1}}-\frac{m_{p}}{T_{\mathrm{em}}}\left(D_{\mathrm{obs}} \lambda_{\mathrm{obs}}+\frac{\sigma_{\mathrm{obs}_{k}}-\sigma_{\mathrm{obs}_{k-1}}}{T_{s}}\right) .
$$

Here, $u_{c}$ is the compensated control input to the system. The positive feedback by input $u_{c}$ forces the system to behave closely toward the ideal system having nominal parameters. However, in reality, there is also some amount of difference between the real disturbance and the estimated disturbances. 


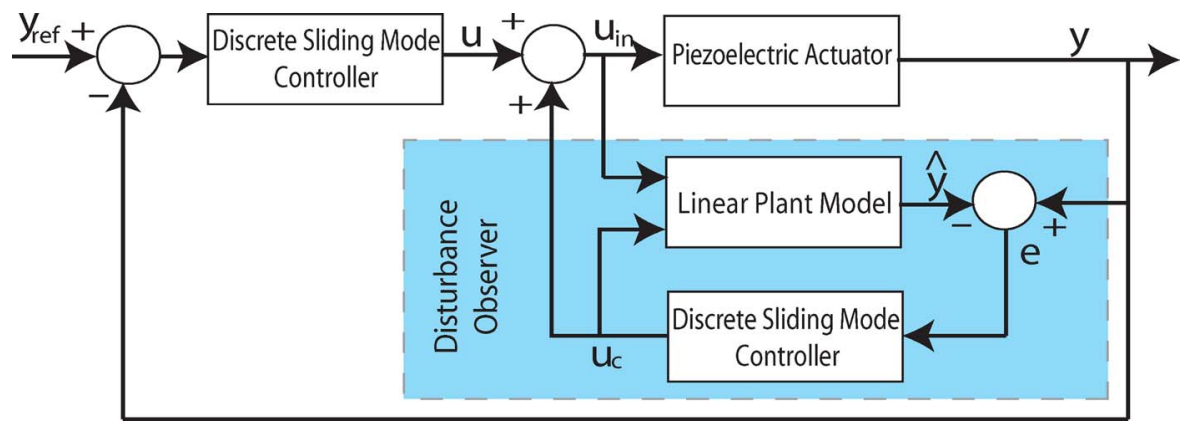

Fig. 4. Controller and disturbance observer for position control of the PZT actuator.

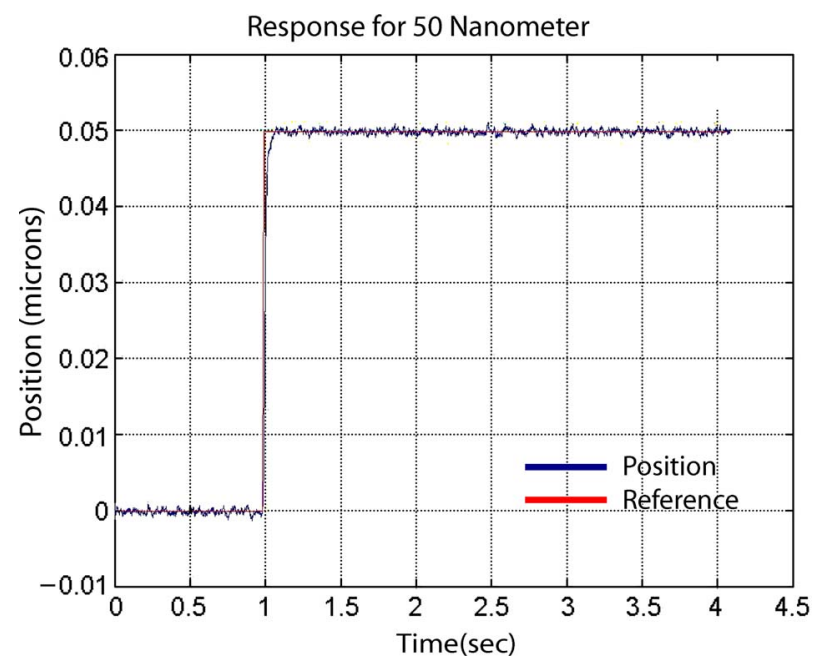

Fig. 5. Position response for a reference of $50 \mathrm{~nm}$ [20].

\section{Experimental Validation of Position Control}

In order to verify the performance of the discrete-time sliding-mode controller along with the disturbance observer, smooth-step inputs are applied to one of the piezostages, and the response is drawn in Fig. 5, which represents the step response for position reference of $50 \mathrm{~nm}$. The rise time and steady state error are $23 \mathrm{~ms}$ and $2 \%$, respectively. An overshoot behavior is not observed, and operation with no overshoot is the foremost requirement for micromanipulation applications since an overshoot may result in damage to the probe or particles. However, the system suffers from noise coming from the measurement devices, which shows up in the steady-state plots.

Fig. 6 shows the response for the trapezoidal input with a height of $0.5 \mu \mathrm{m}$, and the result shows that it precisely follows the reference position and that tracking error is found to be less than $10 \mathrm{~nm}$. Fig. 7 shows the position response for the sinusoidal input with an amplitude of $1 \mu \mathrm{m}$ with low frequency of $2 \mathrm{~Hz}$ due to the requirement of slow motion on the slave side. It can clearly be observed that the actual position tracks the reference with high accuracy and that the tracking error is within $\pm 20 \mathrm{~nm}$. These experimental results suggest that the proposed controller, along with the disturbance observer, produces acceptable results for positioning with very high precision.

\section{Scaled Bilateral Teleoperation}

In micromanipulation applications, scaled bilateral control is used for teleoperation where the master/human is not able to ac-
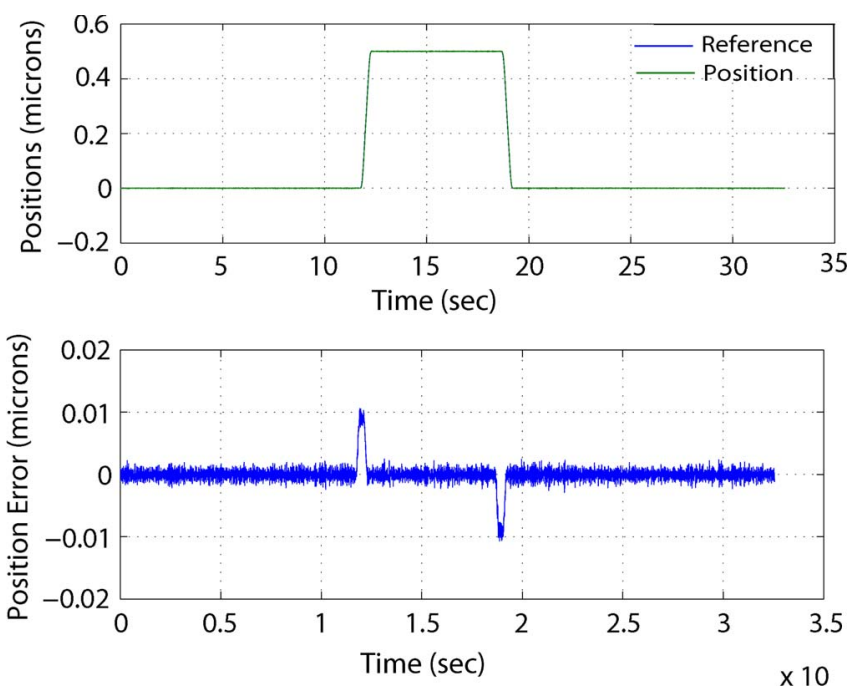

Fig. 6. Position response for a trapezoidal reference.
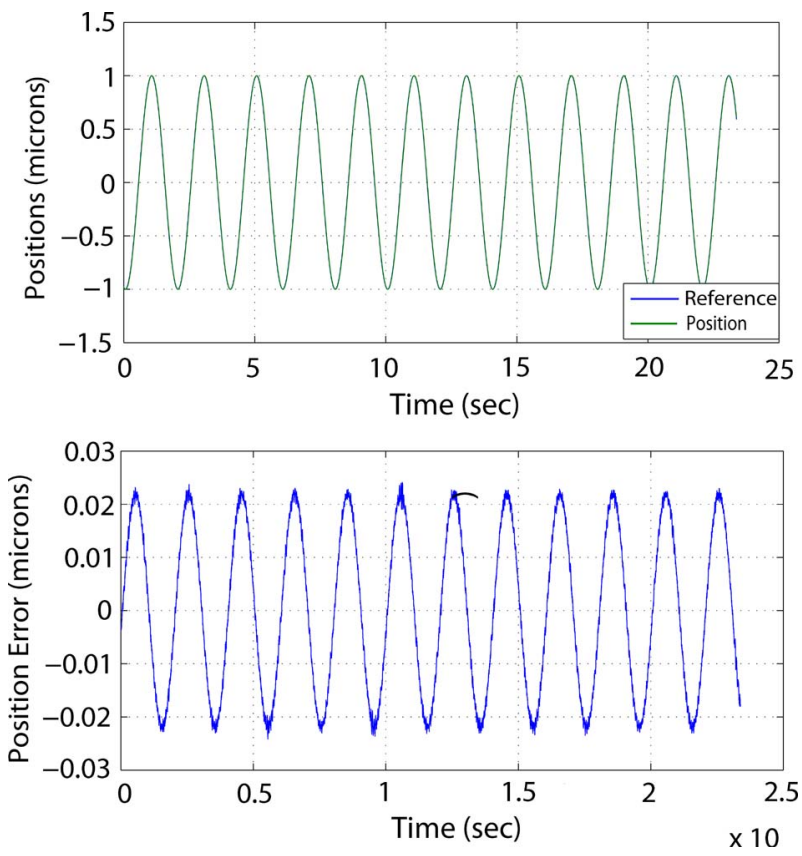

Fig. 7. Position response for a sinusoidal reference for $1-\mu \mathrm{m}$ amplitude.

cess the micro environment on the slave side. Since the master and the slave are working on the macroscale and microscale, respectively, it is indispensable to use a general bilateral controller to scale the position and forces between two sides for 


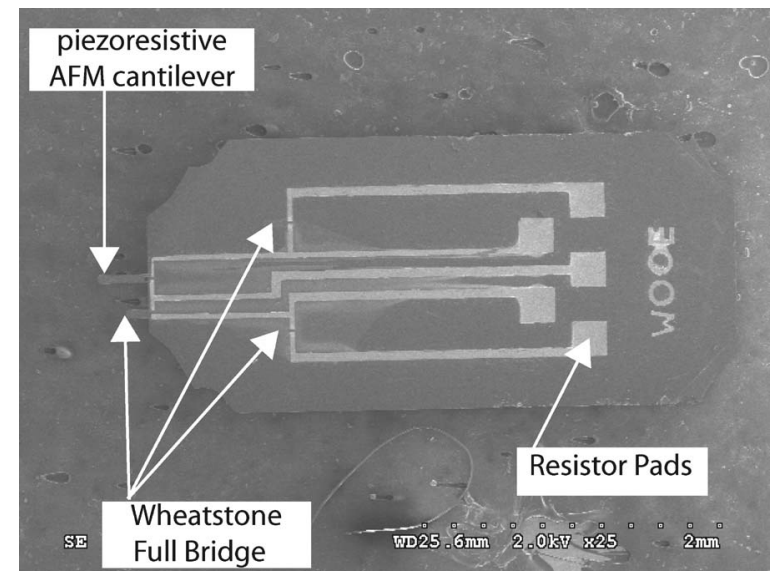

Fig. 8. Piezoresistive AFM cantilever with built-in Wheatstone bridge.

extensive capability. In other words, the position information from the master side is scaled down to the slave, and the force information from the slave side is scaled up to the master.

\section{A. Schematic of Telemicromanipulation Setup}

The complete bilateral structure is shown in Fig. 1, comprising the master and slave sides. A piezostage on the slave side is required to track the master's position, as dictated by the operator using a discrete sliding-mode controller structure, as discussed in the previous section. The $1 D$ force of interaction with the environment, generated by the piezoresistive cantilever, on the slave side is transferred to the master as a force opposing its motion, therefore causing a "feeling" of the environment by the operator. The conformity of this feeling with the real forces is called "transparency." Transparency is crucial for micromanipulation/nanomanipulation applications for stability of the overall system. Furthermore, for microsystem applications, position and forces should be scaled in order to adjust to the operator requirements. The position of the master manipulator, scaled by a factor $\alpha$, is used as a position reference for the slave manipulator, while the calculated force due to contact with the environment, scaled by a factor $\beta$, is fed back to the operator through the master manipulator.

\section{B. Force Sensing Using Piezoresistive AFM Cantilever}

A commercially available piezoresistive microcantilever from Applied NanoStructures, Inc. with an integrated built-in Wheatstone and strain gauge is utilized as the force sensor, as shown in Fig. 8. As the force is applied at the free end of the cantilever, the change of strain gauge resistance takes place, depending on deflection. The amount of deflection is measured by a Wheatstone bridge that provides an analog voltage output and amplified by the amplifier, as shown in Fig. 1 .

To compensate the offset caused by the built-in cantilever resistance value, one of the passive resistors in the Wheatstone bridge is connected to a potentiometer. The amplified voltage corresponding to the forces is then sent to the data acquisition card, and the force is calculated using Hooke's law $F=K_{c} \times$ $z$, where $K_{c}$ is the known spring constant of $0.3603 \mathrm{~N} / \mathrm{m}$ and $z$ is the amount of cantilever deflection. The spring constant
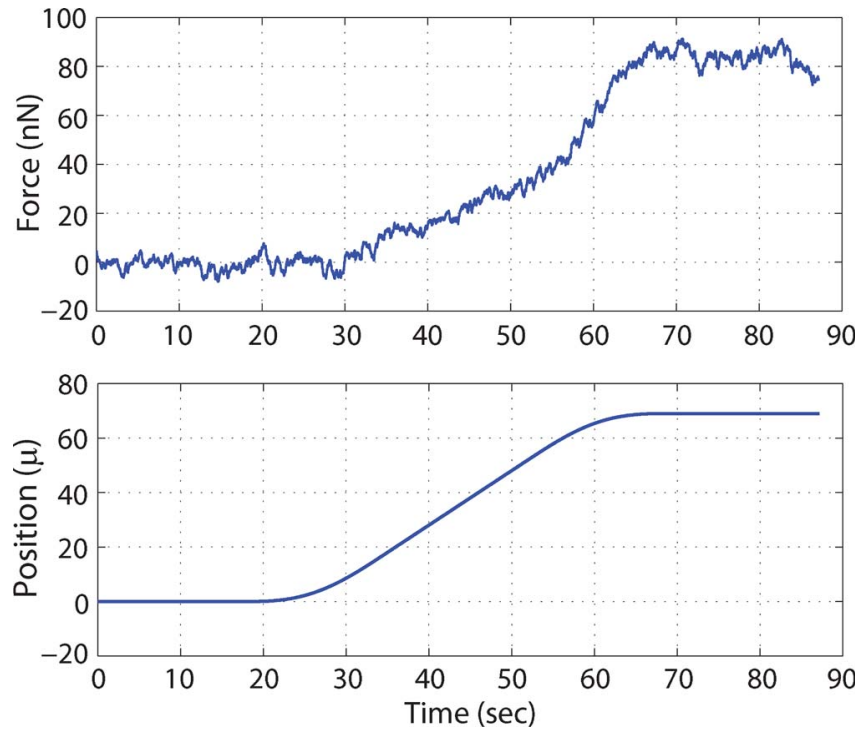

Fig. 9. Force for smooth-step position reference.

is calculated by considering a linear beam equation and verified via a natural frequency test using an AFM [21]. The linear beam equation is represented as $K_{c}=3 E I / L 3$, where $E$ represents the modulus of elasticity (190 GPa for silicon) and $I$ denotes the moment of inertia for a bending beam calculated as $I=$ $b h^{3} / 12$, with $b$ and $h$ representing the width and height of the microcantilever (50 and $1.6 \mu \mathrm{m})$, respectively. The value of $I$ is calculated as $17.067 \times 10^{-24} \mathrm{~m}^{4}$.

The cantilever is mounted on the three-axis closed-loop stages, and the interaction (contact and noncontact) forces between the tip and the glass slide are measured. The movement of the cantilever is selected to be perpendicular to the plane of the optical axis in order to achieve better visibility of the distance between the cantilever and the glass slide. Since the displacement range of the $x$-axis of the closed-loop stage is $100 \mu \mathrm{m}$, the glass slide is brought within the range using open-loop manual PZT axes. Finally, the change of the resistance is converted to change in voltage (millivolt range) using the builtin full bridge along with a potentiometer for offset calibration and sending to the to amplifier to generate $\pm 10 \mathrm{~V}$.

\section{Experimental Validation of Force Sensing}

In order to verify the force sensing, the piezoresistive AFM cantilever is made to interact with the glass slide, and Fig. 9 shows the attractive forces between the tip and the glass slide [22]. A decreasing distance between the tip and the glass slide corresponds to an increase in the position of PZT along the same axis. It can be observed that, as the distance between the tip and the glass slide decreases, attractive forces increase. The first part of the graph is dominated by electrostatic forces, while the remaining part is dominated by van der Waals forces. The change in slope of the force measurement plot corresponding to these two regions can be observed in Fig. 9.

In order to verify the force measurement, theoretical values of the pull-off force (breaking load during the withdrawal of the tip) between the silicon tip and the glass surface are compared with the experimental results. In the case of the interaction 


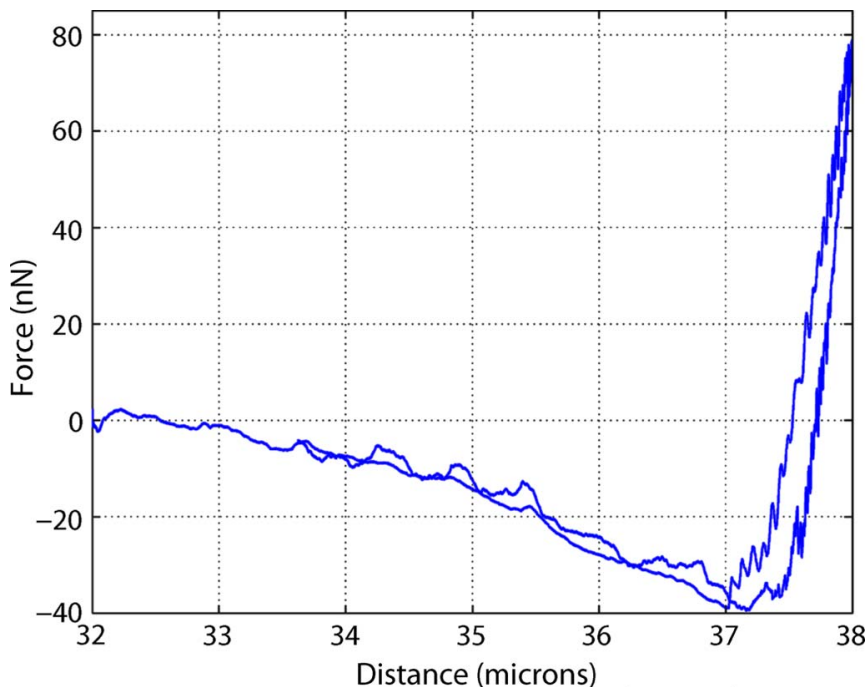

Fig. 10. Forces of interaction between the silicon tip and the glass slide.

between a spherical tip and a planar surface, the interaction force can be approximated by the Dugdale model [23] as

$$
F_{\text {pull-off }}=\left(\frac{7}{4}-\frac{1}{4} \frac{4.04 \lambda^{\frac{1}{4}}-1}{4.04 \lambda^{\frac{1}{4}}+1}\right) \pi W R
$$

where $W$ is the work of adhesion between the two media, $R$ is the radius of the sphere, and $\lambda$ is a coefficient, which can be used to choose the most appropriate contact model for a given case [24]. Using the interfacial energy, the pull-off force can be calculated for $\lambda=0.54$ according to the Dugdale model as $39.43 \mathrm{nN}$ [25], [26]. Fig. 10 shows the experimentally determined pull-off force that is close to $40 \mathrm{nN}$, indicating a close match between the theoretically and experimentally determined values.

\section{Position/Force Tracking of Master and Slave}

In order to attain "full" transparency, it is inevitable that the slave precisely tracks the master position, and simultaneously, the slave also transmits the interaction force from the environment to the master. In our case, the force transmitted by the piezoresistive cantilever is in single dimension.

\section{A. Scaling of Position/Force Information}

Since the master and the slave side reside on the macroscale and the microscale, respectively, it is very vital to appropriately choose the scaling factor in order to attain optimum performance. In ideal condition, the steady-state condition of the bilateral controller should be

$$
\begin{aligned}
x_{s} & =\alpha x_{m} \\
F_{m} & =\beta F_{s}
\end{aligned}
$$

where $\alpha$ and $\beta$ represent the position and force scaling; $x_{m}$ and $x_{s}$ denote the master and slave positions; and $F_{m}$ and $F_{s}$ stand for the master and slave forces, respectively. To be able to meaningfully interact with the micro environment, positions and forces are scaled to match the operator requirements.
In the first and second experiments, scaling factors of $\alpha=$ $0.027 \mu \mathrm{m} / \mathrm{deg}$ and $\beta=0.00366 \mathrm{~N} / \mathrm{nN}$ are used, i.e., an angular displacement of $1 \mathrm{deg}$ on the master side corresponds to a linear displacement of $0.027 \mu \mathrm{m}$ on the slave side, and a force of $0.00366 \mathrm{nN}$ on the slave side corresponds to a force of $1 \mathrm{~N}$ on the master side. The objective of these experiments is to provide very fine motion on the slave side for a relatively larger displacement on the master side, so $\alpha$ is selected according to this objective. Then, the corresponding forces/torques for each amount of displacement were compared for the selection of $\beta$, keeping in mind that the de servo on the master side has low torques.

\section{B. Experimental Validation of Position/Force Tracking}

Fig. 11 shows the experimental results for position tracking along with the tracking error of the master and slave systems. It can clearly be seen that, under different references, the slave tracks the master position with high accuracy. This position tracking performance is acceptable for precisely positioning the microcantilever.

Fig. 12 shows the force tracking between the master and the slave along with the tracking error. It can clearly be observed from the figure that the master tracks the slave force precisely and that the tracking error is found to be within $\pm 20 \mathrm{nN}$. Thus, it can clearly be concluded that using a discrete SMC structure along with a disturbance observer yields very precise position tracking. Force tracking also confirms the transparency between the master and the slave.

\section{CONCLUSION}

In this paper, the design of a discrete-time sliding-mode controller based on Lyapunov theory was presented. A robust disturbance observer based on sliding-mode control was presented and applied to a piezostage by considering all the nonlinearities present in the system as lumped disturbance. A linear model of a piezostage was used with nominal parameters and employed to compensate the disturbance acting on the system in order to achieve nanoscale accuracy. The effectiveness of the controller and disturbance observer was verified in terms of closed-loop position performance. The results show that the proposed controller structure produced good experimental results eliminating any chattering motion but the influence of sensing noise in the high frequency range, effect steady-state position of the system and forces an oscillatory behavior.

The aforementioned discrete-time sliding-mode controller has been applied in the bilateral structure for the custom-built telemicromanipulation setup. A piezoresistive AFM microcantilever with a built-in Wheatstone bridge was utilized to achieve the nanonewton-level interaction forces between the cantilever probe tip and the glass surface. Experimental results are compared to the theoretical estimates of the changes in attractive forces as a function of decreasing distance and of the pull-off force between a silicon tip and a glass surface. Good agreement among the experimental data and the theoretical estimates has been demonstrated. Force/position tracking between the master and the slave has clearly been demonstrated after necessary 

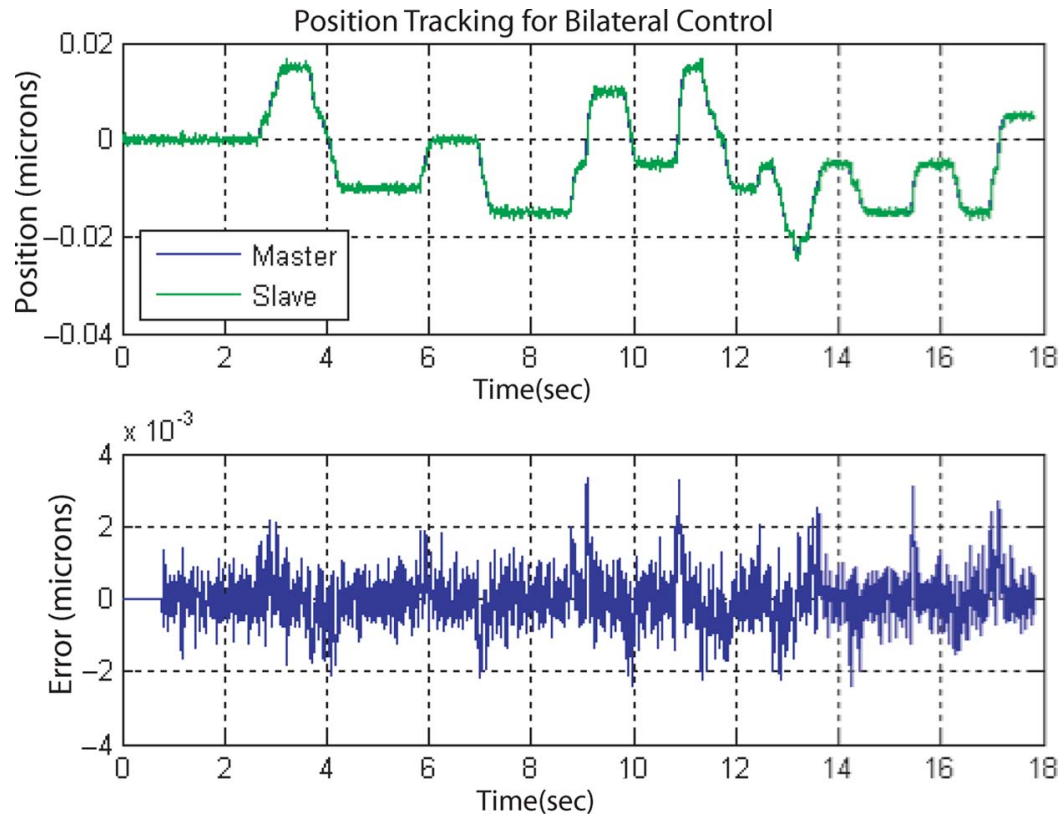

Fig. 11. Position tracking of the bilateral controller for zigzag motion with 20-nm amplitude.
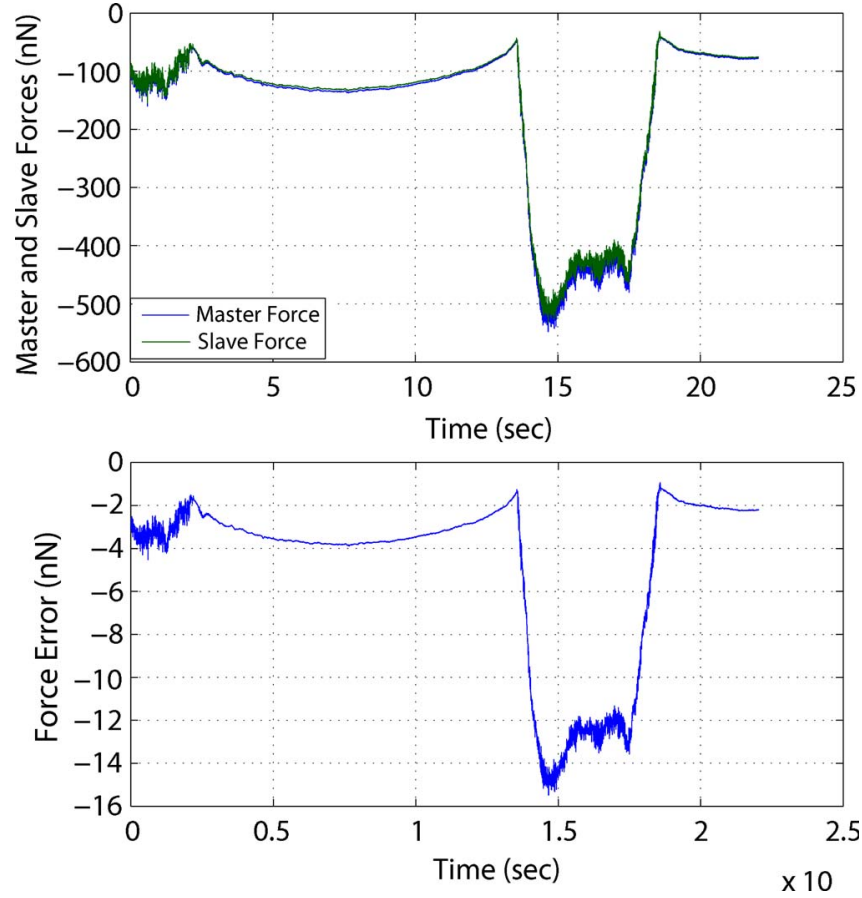

Fig. 12. Force tracking of the bilateral controller and tracking error.

scaling. It is clearly demonstrated that the slave position tracked the master position with high precision and that the master felt the interaction forces in one dimension between the slave and the environment.

\section{REFERENCES}

[1] G. Yang, J. A. Gaines, and B. Nelson, "Optomechatronic design of microassembly systems for manufacturing hybrid microsystems," IEEE Trans. Ind. Electron., vol. 52, no. 4, pp. 1013-1023, Aug. 2005.

[2] D. O. Popa and H. E. Stephanou, "Micro and meso scale robotic assembly," in Proc. WTEC Workshop: Rev. U.S. Res. Robot., 2004, pp. 1-20.
[3] C. D. Onal, "Bilateral control-A sliding mode control approach," M.S. thesis, Sabanci Univ., Istanbul, Turkey, 2005.

[4] T. Shimono, S. Katsura, and K. Ohnishi, "Abstraction and reproduction of force sensation from real environment by bilateral control," IEEE Trans. Ind. Electron., vol. 54, no. 2, pp. 907-918, Apr. 2007.

[5] S. Katsura, Y. Matsumoto, and K. Ohnishi, "Realization of law of action and reaction by multilateral control," IEEE Trans. Ind. Electron., vol. 52, no. 5, pp. 1196-1205, Oct. 2005.

[6] S. Katsura and K. Ohnishi, "A realization of haptic training system by multilateral control," IEEE Trans. Ind. Electron., vol. 53, no. 6, pp. 19351942, Dec. 2006.

[7] M. Sitti and H. Hashimoto, "Teleoperated touch feedback from the surfaces at the nanoscale: Modeling and experiments," IEEE/ASME Trans. Mechatronics, vol. 8, no. 2, pp. 287-298, Jun. 2003.

[8] T. Tsuji, K. Natori, and K. Ohnishi, "A controller design method of bilateral control system," in Proc. Eur. Power Electron. Power Electron. Motion Control Conf., 2004, vol. 4, pp. 123-128.

[9] L. Huijun and S. Aiguo, "Virtual-environment modeling and correction for force-reflecting teleoperation with time delay," IEEE Trans. Ind. Electron., vol. 54, no. 2, pp. 1227-1233, Apr. 2007.

[10] R. Anderson and M. Spong, "Bilateral control of teleoperators with time delay," IEEE Trans. Autom. Control, vol. 34, no. 5, pp. 494-501, May 1989.

[11] B. M. Chen, T. H. Lee, C.-C. Hang, Y. Guo, and S. Weerasooriya, "An $\mathrm{h}$ infinity almost disturbance decoupling robust controller design for a piezoelectric bimorph actuator with hysteresis," IEEE Trans. Control Syst. Technol., vol. 7, no. 2, pp. 160-174, Mar. 1999.

[12] R. Banning, W. L. de Koning, and J. M. T. A. Adriaens, "Modeling piezoelectric actuators," IEEE/ASME Trans. Mechatronics, vol. 5, no. 4, pp. 331-341, Dec. 2000.

[13] K. Young, V. Utkin, and U. Ozguner, "A control engineer's guide to sliding mode control," IEEE Trans. Control Syst. Technol., vol. 7, no. 3, pp. 328342, May 1999.

[14] V. I. Utkin, "Variable structure systems with sliding modes," IEEE Trans. Autom. Control, vol. AC-22, no. 2, pp. 212-222, Apr. 1977.

[15] A. Sabanovic, M. Elitas, and K. Ohnishi, "Sliding modes in constrained systems control," IEEE Trans. Ind. Electron., vol. 55, no. 9, pp. 33323339, Sep. 2008.

[16] K. Abidi and A. Sabanovic, "Sliding-mode control for high-precision motion of a piezostage," IEEE Trans. Ind. Electron., vol. 54, no. 1, pp. 629637, Feb. 2007

[17] C. Pawashe and M. Sitti, "Two-dimensional vision based autonomous microparticle manipulation using a nanoprobe," J. Micromechatronics, vol. 3, no. 3-5, pp. 285-306, 2006.

[18] S. V. Drakunov and V. Utkin, "A semigroup approach to discrete-time sliding modes," in Proc. Amer. Control Conf., 1995, pp. 1314-1317.

[19] M. Goldfarb and N. Celanovic, "Modeling piezoelectric stack actuators for control of micromanipulation," IEEE Control Syst. Mag., vol. 17, no. 3, pp. 69-79, Jun. 1997. 
[20] S. Khan, M. Elitas, E. D. Kunt, and A. Sabanovic, "Discrete sliding mode control of piezo actuator in nano-scale range," in Proc. IEEE ICIT, 2006, pp. 1454-1459.

[21] J. Hutter and J. Bechhoefer, "Calibration of atomic-force microscope tips," Rev. Sci. Instrum., vol. 64, no. 7, pp. 1868-1873, Jul. 1993.

[22] S. Khan, A. O. Nergiz, A. Sabanovic, and V. Patoglu, "Development of a micromanipulation system with force sensing," in Proc. IEEE/IROS Int. Conf. Intell. Robots Syst., 2007, pp. 285-290.

[23] K. L. Johnson, "A continuum mechanics model of adhesion and friction in a single asperity contact," in Micro-Nanotribology and its Applications. Norwell, MA: Kluwer, 1997.

[24] D. Maugis, "Adhesion of spheres: The JKR-DMT transition model using a Dugdale model," J. Colloid Interface Sci., vol. 150, no. 1, pp. 243-269, Apr. 1992.

[25] M. Sitti and R. Fearing, "Synthetic gecko foot-hair micro-nanostructures as dry adhesives," J. Adhesion Sci. Technol., vol. 17, no. 8, pp. 1055-1073, 2003.

[26] P. Rougeot, S. Regnier, and N. Chaillet, "Force analysis for micromanipulation," in Proc. IEEE CIRA, 2005, pp. 105-110.

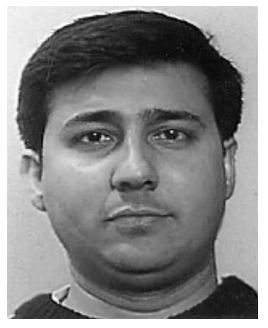

Shahzad Khan (M'07) received the B.Sc. degree in physics and the M.Sc. degree in electronics from Kanpur University, Kanpur, India, in 1999 and 2001, respectively, and the Ph.D. degree in mechatronics programme from Sabanci University, Istanbul, Turkey, in 2008.

$\mathrm{He}$ was a Research Associate with the Centre for Mechatronics, Indian Institute of Technology, Kanpur, until August 2004. Since February 2008, he has been a Postdoctoral Researcher with Department of Precision and Microsystems Engineering, Delft University of Technology, Delft, The Netherlands. His research interest include haptics, high-precision motion control, and microassembly techniques.

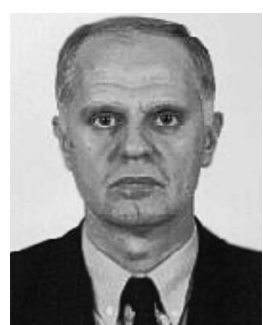

Asif Sabanovic (M'85-SM'03) received the B.S., M.S., and Dr.Sci. degrees in electrical engineering from the University of Sarajevo, Sarajevo, Bosnia and Herzegovina, in 1970, 1975, and 1979, respectively.

Previously, he was with the University of Sarajevo. He was a Visiting Professor with the California Institute of Technology, Pasadena; Keio University, Yokohama, Japan; and Yamaguchi University, Yamaguchi, Japan. He was the Head of the CAD/CAM and Robotics Department, Marmara Research Center (MAM), Scientific and Technological Research Council of Turkey (TUBITAK), Turkey. He is currently with Sabanci University, Istanbul, Turkey. His fields of research interest include power electronics, sliding-mode control, motion control, and mechatronics.

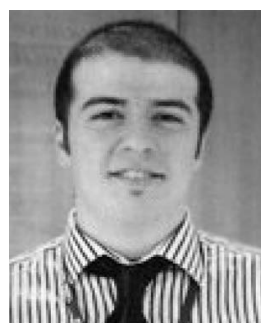

Ahmet Ozcan Nergiz received the B.S. and M.Sc. degrees in mechatronics engineering from Sabanci University, Istanbul, Turkey, in 2006 and 2009, respectively. $\mathrm{He}$ is currently working toward the Ph.D. degree at Northeastern University, Boston, MA.

Since 2008, he has been with Northeastern University, where he is currently a Research Assistant with the NSF Center for High-Rate Nanomanufacturing. His current research includes nanomanufacturing of devices using nanotubes and optimization of their dielectrophoretic assembly conditions. 